\title{
De-noising Spacecraft Attitude and Rate Data using DWT
}

\author{
B. Ravi Sankar \\ Scientist \\ MPAD/MDG, ISRO Satellite Centre, Bangalore, \\ INDIA.
}

\author{
S. Alamelu Mangai \\ Dept. of Computer Science, \\ Dr. Ambedkar Institute of Management Studies, \\ Bangalore, INDIA.
}

\begin{abstract}
In this paper, de-noising of satellite attitude and rate data using discrete wavelet transform is presented. The mission objective of any remote sensing satellite is to produce an image. The image is taken on board the spacecraft, and then downloaded using a Radio Frequency link. The processing of the image is done at ground. The attitude quaternion and body rates are part of the down linked data. These data had to be pre-processed to remove the noise. Conventional methods employ taking the Fourier transform and removing the high frequency component through filtering. The de-noising of attitude and rate data is important to make the final data product such as image smoother.
\end{abstract}

\section{General Terms}

Spacecraft, attitude, body rates.

\section{Keywords}

Satellite image, Satellite Data Product, attitude quaternion denoising, body rates denoising, Discrete Wavelet Transform, Remote Sensing satellite.

\section{INTRODUCTION}

Spacecraft attitude [1] and body rate play an important role in obtaining the final image or the so called data product. Along with payload data, spacecraft attitude and body rates also will be down linked. Before processing the payload data which is the actual image, the attitude and body rates have to be denoised. Conventional methods use taking the FFT and removing the high frequency component which is presumed as noise. In this paper we propose a novel method for removing the noise based on Discrete Wavelet Transform (DWT). At present wavelets are used to compress the image data of a spacecraft.

The paper is organized as follows. Section 2 describes the terminology and provides the detail of the satellite used in the analysis. An introduction to wavelet transform is given in section 3. In section 4, the spacecraft data is analyzed based on discrete wavelet transform using MATLAB. Section 5 concludes this paper.

\section{TERMINOLGY}

\subsection{Attitude}

Attitude [1] is defined as the orientation of some orthogonal Cartesian axes with respect to some reference chosen axes. Attitude is always represented using quaternions in spacecrafts. Figure 1 shows the attitude geometry. In Figure 1 , reference frame is represented by XYZ and spacecraft body frame is represented as YRP.

\subsection{Rates}

Rate [1] of axis is defined as change in angle of a chosen axis with respect to reference axis per second. Always rates are given in body frame i.e. yaw roll pitch frame, at least for the purpose of processing the payload data.

\subsection{Introduction to the SMART-1}

SMART-1 was a Swedish-designed | European Space Agency satellite that orbited around the Moon. It was launched on September 27, 2003 at 23:14 UTC from the Guiana Space Centre in Kourou, French Guiana. "SMART-1" stands for Small Missions for Advanced Research in Technology-1. On September 3, 2006 (05:42 UTC), SMART-1 was deliberately crashed into the Moon's surface, ending its mission. The satellite data is available in public domain through ESA website. The data from the SMART-1 satellite is used in this analysis. The source data used for analyzing are shown in figure 2 and figure 3 . The data corresponds to day number 272 of the year 2004 and the time interval is given below.

\section{Start Time [UTCDOY]: 2004/272 00:00:19.001 \\ End Time [UTCDOY]: 2004/272 00:20:20.378}

Time-step [DDD HH:MM:SS.SSS]: 000 00:00:00.128

Where UTCDOY stands for Universal Time Coordinate Day Of Year. DDD stands for day number. HH stands for hour number. MM stands for minute number. SS stands for second number. SSS stands for milli-second number and it is the sampling time.

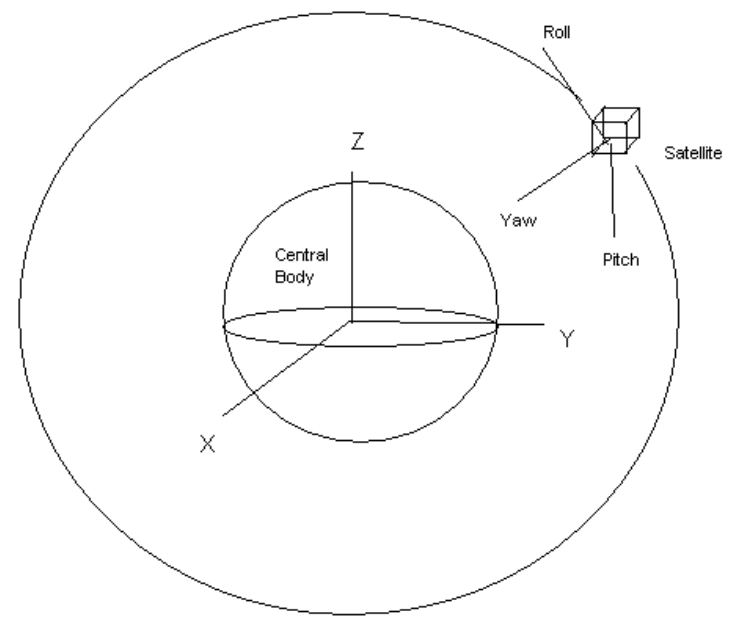

Figure 1: Geometry of Spacecraft and Reference Body 


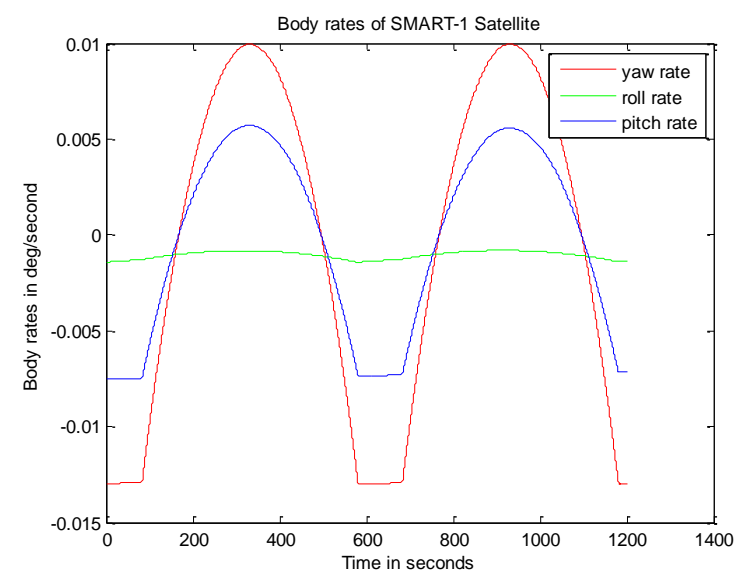

Figure 2: SMART-1 Spacecraft Body Rates in Body Frame

\section{DISCRETE WAVELET TRANSFORM}

Wavelets are mathematical functions that analyze data according to scale or resolutions [2]. They aid in studying a signal in different windows or at different resolutions. For instance, if the signal is viewed in a large window, gross features can be noticed, but if viewed in a small window, only small features can be noticed. Wavelets provide some advantages over Fourier transforms. For example, they do a good job in approximating signals with sharp spikes or signals having discontinuities. Wavelets can also model speech, music, video and non-stationary stochastic signals. Wavelets can be used in applications such as image compression, turbulence, human vision, radar, earthquake prediction, etc. [3]. DWT is representation of wavelets in digital computers.

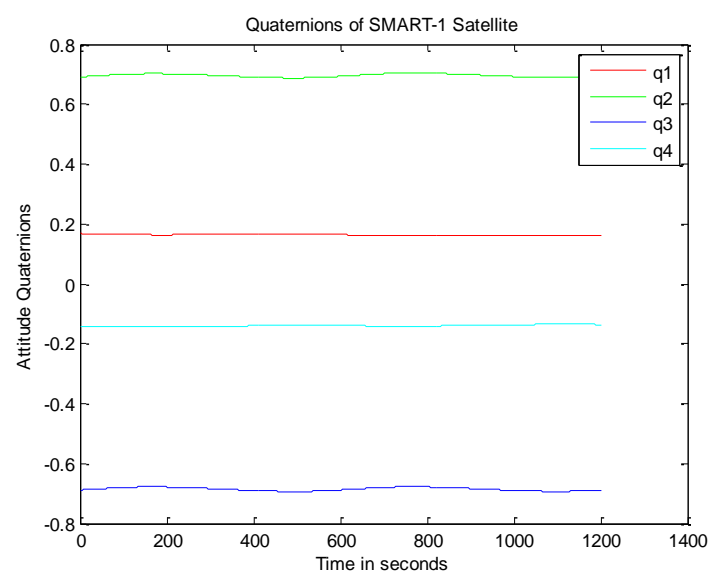

Figure 3: SMART-1 Spacecraft Attitude Quaternion in ECI Frame

\section{ANALYSIS USING DWT}

Whatever the data down linked from satellite is a discrete time signal consisting of a vector of numbers. The signals were analyzed with various mother wavelets and finally found Haar wavelet [2] is suitable for this analysis purely based on trial and error. The original signals were decomposed at a level of 12 and threshold (using rigorous sure threshold) the coefficients, for noise removal [3]. The noise residuals were reconstructed and found the important characteristics of the noise that are tabled in table 1 . Figure 4 shows the yaw rate decomposed at level 12 using Haar wavelet and a similar procedure was adopted for rest of the parameters. All the rest of the figures show the autocorrelation and FFT spectrum for all the seven noise residuals.

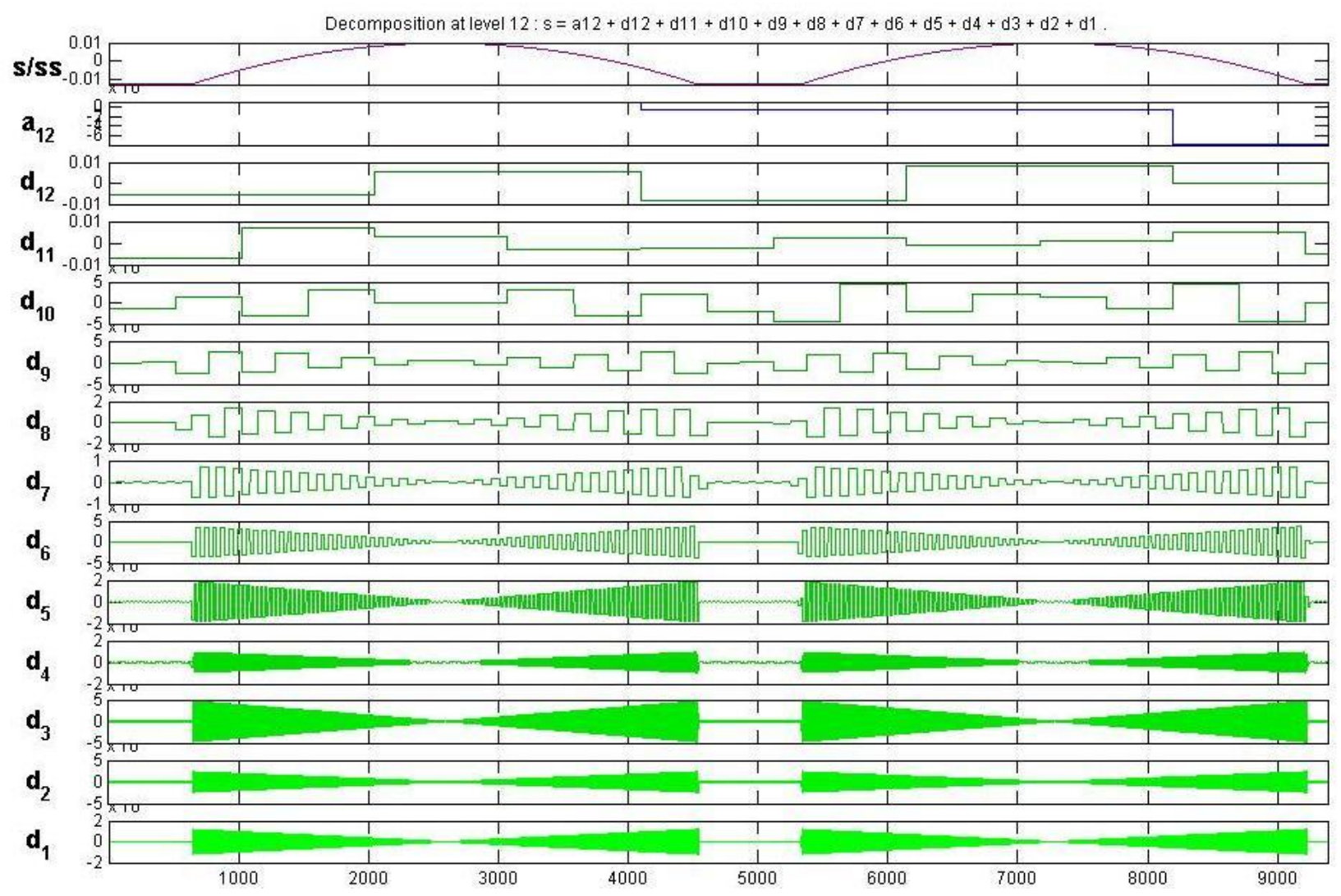

Figure 4: Haar Wavelet Decomposition of Yaw rate at Level 12 

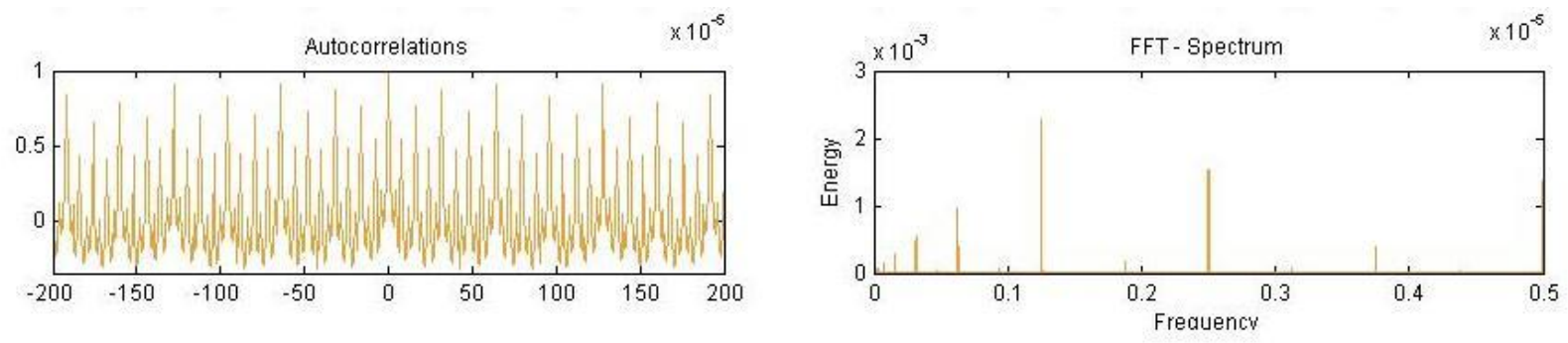

Figure 5: Autocorrelation and FFT Spectrum of Noise residuals of Yaw rate
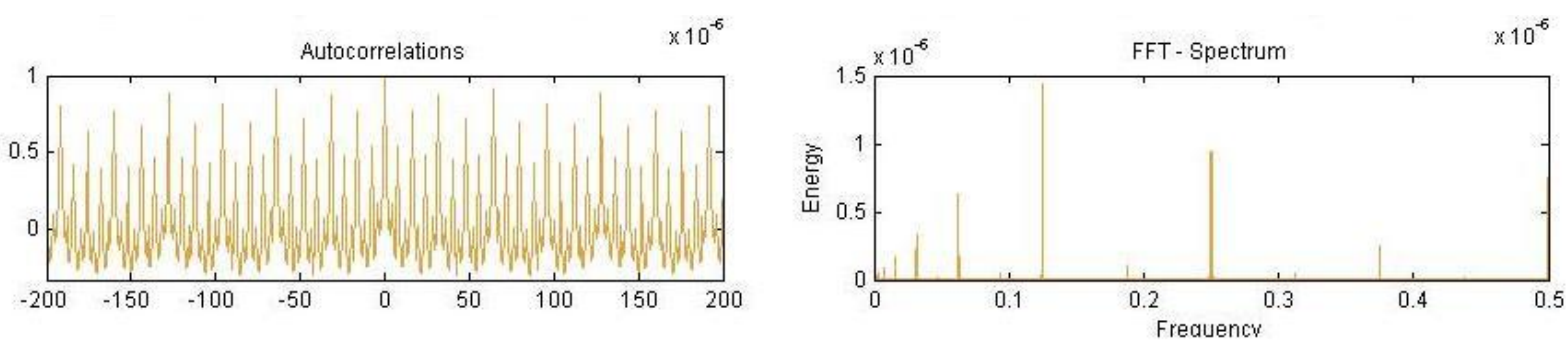

Figure 6: Autocorrelation and FFT Spectrum of Noise residuals of Roll rate
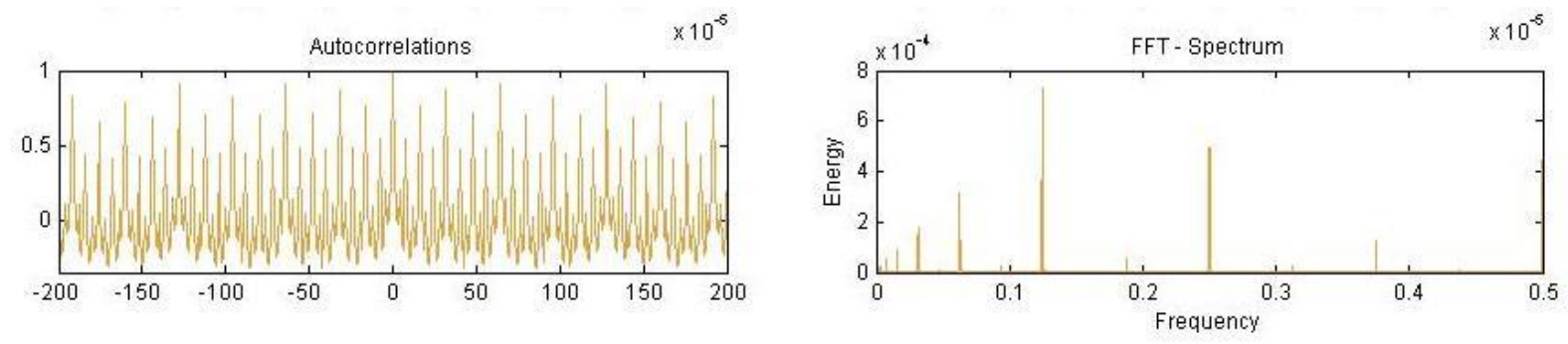

Figure 7: Autocorrelation and FFT Spectrum of Noise residuals of Pitch rate
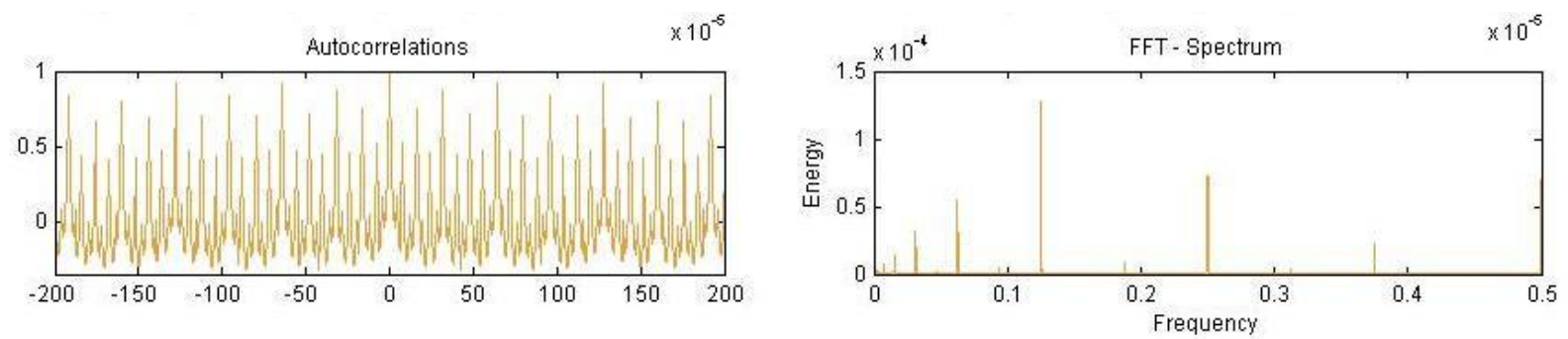

Figure 8: Autocorrelation and FFT Spectrum of Noise residuals of quaternion-1
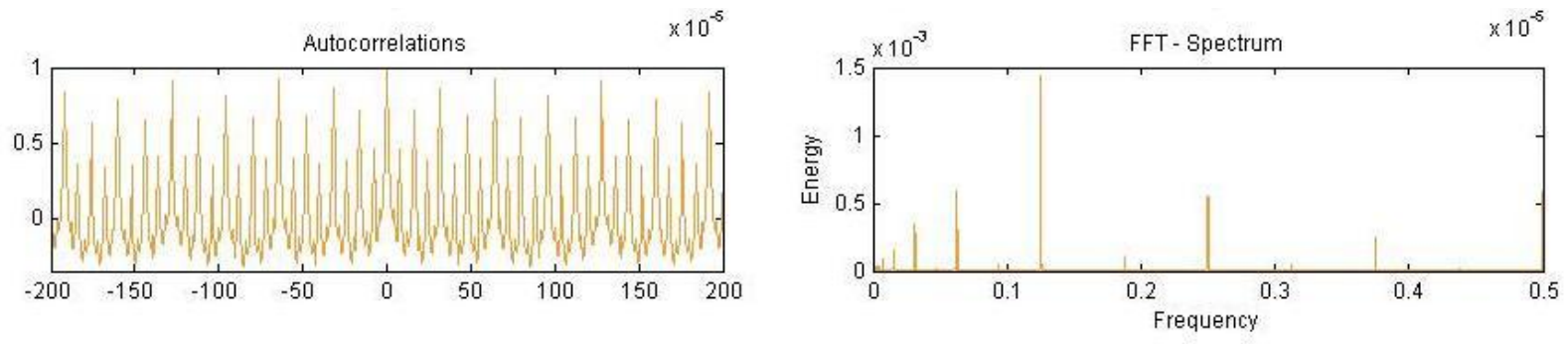

Figure 9: Autocorrelation and FFT Spectrum of Noise residuals of quaternion-2 

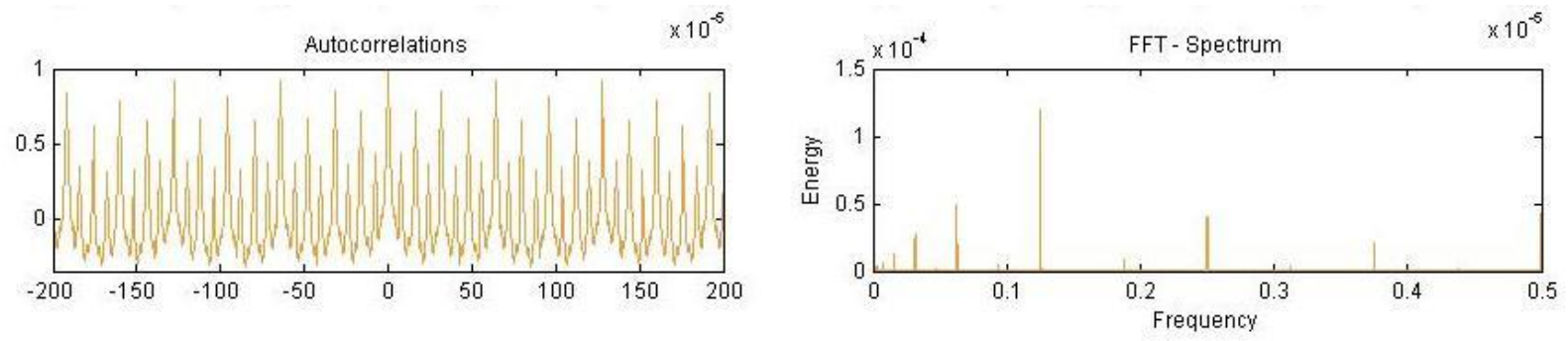

Figure 10: Autocorrelation and FFT Spectrum of Noise residuals of quaternion-3
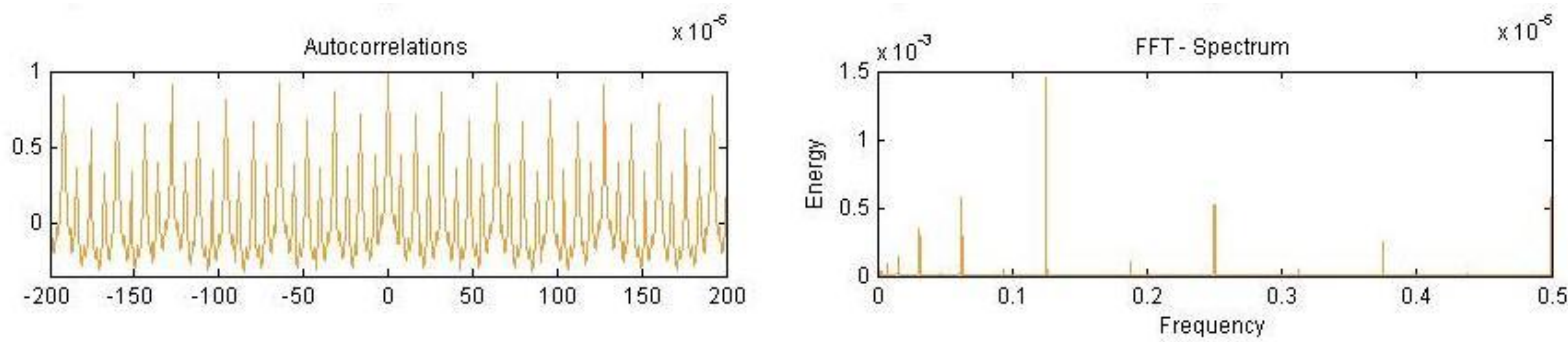

Figure 11: Autocorrelation and FFT Spectrum of Noise residuals of quaternion-4

Table 1: Important parameters of all noise residuals.

\begin{tabular}{|l|l|l|l|l|l|l|l|}
\hline Parameter & Yaw rate & Roll Rate & Pitch Rate & Q1 & Q2 & Q3 & Q4 \\
\hline Mean & $1.08 \mathrm{e}-007$ & $9.276 \mathrm{e}-010$ & $5.139 \mathrm{e}-008$ & $2.709 \mathrm{e}-008$ & $-9.502 \mathrm{e}-008$ & $-9.625 \mathrm{e}-008$ & $2.981 \mathrm{e}-008$ \\
\hline Median & $-1.532 \mathrm{e}-006$ & $1.737 \mathrm{e}-008$ & $-5.239 \mathrm{e}-007$ & $1.684 \mathrm{e}-009$ & $-8.433 \mathrm{e}-008$ & $-6.626 \mathrm{e}-008$ & $-1.192 \mathrm{e}-010$ \\
\hline Mode & $-3.13 \mathrm{e}-006$ & $2.757 \mathrm{e}-007$ & $-1.999 \mathrm{e}-006$ & $-1.444 \mathrm{e}-007$ & $3.115 \mathrm{e}-007$ & $-1.196 \mathrm{e}-006$ & $-7.765 \mathrm{e}-007$ \\
\hline Maximum & $7.614 \mathrm{e}-005$ & $1.715 \mathrm{e}-006$ & $4.247 \mathrm{e}-005$ & $1.704 \mathrm{e}-005$ & $5.157 \mathrm{e}-005$ & $5.218 \mathrm{e}-005$ & $1.567 \mathrm{e}-005$ \\
\hline Minimum & $-7.929 \mathrm{e}-005$ & $-1.796 \mathrm{e}-006$ & $-4.473 \mathrm{e}-005$ & $-1.665 \mathrm{e}-005$ & $-5.304 \mathrm{e}-005$ & $-5.248 \mathrm{e}-005$ & $-1.536 \mathrm{e}-005$ \\
\hline Range & 0.0001554 & $3.511 \mathrm{e}-006$ & $8.72 \mathrm{e}-005$ & $3.369 \mathrm{e}-005$ & 0.0001046 & 0.0001047 & $3.104 \mathrm{e}-005$ \\
\hline $\begin{array}{l}\text { Standard } \\
\text { Deviation }\end{array}$ & $2.253 \mathrm{e}-005$ & $5.508 \mathrm{e}-007$ & $1.279 \mathrm{e}-005$ & $5.079 \mathrm{e}-006$ & $1.664 \mathrm{e}-005$ & $1.674 \mathrm{e}-005$ & $5.115 \mathrm{e}-006$ \\
\hline $\begin{array}{l}\text { Median } \\
\text { Absolute } \\
\text { Deviation }\end{array}$ & $1.274 \mathrm{e}-005$ & $3.644 \mathrm{e}-007$ & $7.162-006$ & $3.35 \mathrm{e}-006$ & $1.118 \mathrm{e}-005$ & $1.13 \mathrm{e}-005$ & $3.48 \mathrm{e}-006$ \\
\hline $\begin{array}{l}\text { Mean } \\
\text { Absolute } \\
\text { Deviation }\end{array}$ & $1.689 \mathrm{e}-005$ & $4.394 \mathrm{e}-007$ & $9.675 \mathrm{e}-006$ & $4.033 \mathrm{e}-006$ & $1.333 \mathrm{e}-005$ & $1.343 \mathrm{e}-005$ & $4.111 \mathrm{e}-006$ \\
\hline
\end{tabular}

\section{CONCLUSION}

Processing of spacecraft payload data is utmost important thing in the overall mission of the satellite. The objective of the launch vehicle is to put the spacecraft into orbit. The purpose of the spacecraft is its payload. The payload may be a communication transponder or imaging payload. Imaging payload spacecraft's are referred to as commonly remote sensing spacecraft. The product of the remote sensing spacecraft is an image. In order to process the image, one needs finer attitude and body rate data. If the attitude and rate data are finer i.e. free from noise, then the resulting image will be smoother. Thus a novel approach for pre-processing the attitude and rate data is developed in this paper based on discrete wavelet transform. The objective of the spacecraft mission is accomplished, when one gets a good image or data product. But the scope for general public to use the satellite data for de-nosing is limited because no organization is willing to give their data to public. Even in this paper, we have downloaded the attitude quaternions and body rates only because that much data is only allowed. The SMART-1 
mission is a moon pointing mission and the final image will be a map of moon.

In this paper, we have emphasized that if the body attitude quaternion and body rates are preprocessed for noise using DWT, then the resulting image will be of good quality. After the image is processed with the de-noised body rate and quaternion, another round of digital image processing has to be done. In this case two dimensional discrete wavelet transform can be employed to remove any spatial or nonwhite noise from the image. The noise characteristic can be given as a feedback to the control system designer of a spacecraft, so as to enable the designer to design a more efficient control system.

\section{REFERENCES}

[1] J.R.Wertz., Spacecraft Attitude Determination and Control, Springer, 1978.

[2] Amara Graps., An introduction to wavelets, http://www.eecis.udel.edu/ amer/CISC651/IEEEwavelet .pdf

[3] Mallat,S., A Wavelet Tour of Signal Processing. Academic Press, New York, 1999. 